Pacific Journal of Mathematic 


\title{
ON HOMOGENEOUS LINEAR DIFFERENTIAL EQUATIONS WITH ARBITRARY CONSTANT COEFFICIENTS
}

\author{
A. SEIDENBERG
}

Let $K$ be an arbitrary ordinary differential field - for our purposes it is sufficient to consider an arbitrary (algebraic) field $K$ which is converted into a differential field by setting $c^{\prime}=0$ for every $c \in K$. Let $u$ be a differential indeterminate over $K$ and let $u=u_{0}, u_{1}, \cdots$ represent the successive derivatives of $u$. Further, let $c_{0}, \cdots, c_{m}$ be arbitrary constants over the field $K\langle u\rangle=$ $K\left(u_{0}, u_{1}, \cdots\right)$, that is, $m+1$ further indeterminates with which we compute in the usual way, setting $c_{i}^{\prime}=0$. In addition to the ring $R=K\{u\}=K\left[u_{0}, u_{1}, \cdots\right]$, we will also be interested in the rings $R_{t+m}=K\left[u_{0}, u_{1}, \cdots, u_{t+m}\right]$. Theorems referring to some one of these rings $R_{t+m}$ may, if convenient, be regarded as belonging to ordinary, rather than differential, algebra, but we will still apply the operation of differentiation to elements of $R_{t+m}$ (not involving $u_{t+m}$ ). This then amounts to a convenience in writing formulas.

Let $l_{0}=c_{0} u_{0}+\cdots+c_{m} u_{m}$. This element generates a prime differential ideal $\left[l_{0}\right]=\left(l_{0}, l_{1}, \ldots\right)$ in $S=K(c)\{u\}$, where $l_{i}=c_{0} u_{i}+\cdots+c_{m} u_{i+m}$. We are interested in having explicitly a basis for $\left[l_{0}\right] \cap K\{u\}$. If $\Delta(u)$ is the determinant of coefficients of any $m+1$ of the $l_{i}$ regarded as linear forms in the $c_{j}$, then clearly $\Delta(u) \in\left[l_{0}\right] \cap K\{u\}$ and Theorem 2 below asserts that the $\Delta(u)$ obtained from all choices of the $l_{i}$ form the required basis.

Let us confine ourselves to the rings $R_{t+m}$ and $S_{t+m}=K(c)\left[u_{0}, \cdots, u_{t+m}\right]$. In $S_{t+m}$, let $p=\left(l_{0}, \cdots, l_{t}\right)$.

Lemma $1 . p=\left(l_{0}, \cdots, l_{t}\right)$ is an m-dimensional prime ideal in $S_{t+m}$.

Proof. Let $G\left(u_{0}, \cdots, u_{t+m}\right) \in S_{t+m}$. Eliminating successively $u_{t+m}$, $u_{t+m-1}, \cdots, u_{m} \bmod \left(l_{0}, \cdots, l_{t}\right)$, we may write $G\left(u_{0}, \cdots, u_{t+m}\right) \equiv G_{1}\left(u_{0}, \cdots\right.$, $\left.u_{m-1}\right) \bmod \left(l_{0}, \cdots, l_{t}\right)$, where $G_{1} \in S_{t+m}$ is a polynomial in the indicated variables. Moreover, starting with indeterminate values $\xi_{i}$ for $u_{i}, i=0, \cdots, m-1$, we can build up a zero $\left(\xi_{0}, \cdots, \xi_{t+m}\right)$ of $p$ by defining $\xi_{m}$ from the condition

Received December 7, 1953, This paper was written while the author was a Guggenheim Fellow.

Pacific J. Math. 5 (1955), 599.606 
$l_{0}(\xi)=0$, and defining $\xi_{m+i}$ successively from the condition $l_{i}(\xi)=0$. Then $\left(\xi_{0}, \cdots, \xi_{t+m}\right)$ is clearly a general point of $p$, whence $p$ is prime and $m$-dimensional.

Lemma 2. Let $p \cap R_{t+m}=P$; and let $t \geq m-1$. Then $P$ is a $2 m$-dimensional prime ideal in $R_{t+m}$.

Proof. Consider the equations:

$$
\begin{aligned}
& c_{0} \xi_{0}+\cdots+c_{m} \xi_{m}=0 \\
& c_{0} \xi_{1}+\cdots+c_{m} \xi_{1+m}=0 \\
& \vdots \\
& c_{0} \xi_{m-1}+\cdots+c_{m} \xi_{2 m-1}=0
\end{aligned}
$$

From these we are going to solve successively for the $c_{i}, i=0, \cdots, m-1$. Since $\xi_{0} \neq 0$, we can solve for $c_{0}$ and find $c_{0} \in K\left(c_{1}, \cdots, c_{m}, \xi_{0}, \cdots, \xi_{m}\right)$. Suppose in this way, solving successively for the $c_{i}$, we find

$$
c_{0}, \cdots, c_{i} \in K\left(c_{i+1}, \cdots, c_{m}, \xi_{0}, \cdots, \xi_{m+i}\right), \quad i<m-1 .
$$

In fact, assume we have found inductively that

$$
\begin{aligned}
& c_{0}, \cdots, c_{i} \in K\left(\xi_{0}, \cdots, \xi_{2 i+1}\right) \cdot c_{i+1} \\
& \quad+K\left(\xi_{0}, \cdots, \xi_{2 i+2}\right) \cdot c_{i+2}+\cdots+K\left(\xi_{0}, \cdots, \xi_{i+m}\right) \cdot c_{m} .
\end{aligned}
$$

Since

$$
\begin{aligned}
& \text { dt } K\left(c_{0}, \cdots, c_{m}, \xi_{0}, \cdots, \xi_{m+i}\right) / K\left(c_{0}, \cdots, c_{m}\right)=m \text { and } \\
& \text { dt } K\left(c_{0}, \cdots, c_{m}\right) / K=m+1
\end{aligned}
$$

we have

$$
\begin{aligned}
\text { dt } K\left(c_{0}, \cdots, c_{m}, \xi_{0}, \cdots, \xi_{m+i}\right) / K=2 m+1 \\
\quad=\operatorname{dt} K\left(c_{i+1}, \cdots, c_{m}, \xi_{0}, \cdots, \xi_{m+i}\right) / K,
\end{aligned}
$$

where dt stands for "degree of transcendency". From this we see that $\xi_{0}, \cdots$, $\xi_{m+i}$ are algebraically independent over $K$ ( since the set $c_{i+1}, \ldots, \xi_{m+i}$ has 
$2 m+1$ members), in particular they are not zero. The coefficient of $c_{i+1}$ in $l_{i+1}(\xi)$ is $\xi_{2(i+1)}$ plus a term in $K\left(\xi_{0}, \cdots, \xi_{2 i+1}\right)$ arising from $c_{0} \xi_{i+1}+\cdots+$ $c_{i} \xi_{2 i+1}$, and since $i+1<m$, we have $2(i+1)<m+i+1$ and $\xi_{2(i+1)} \notin$ $K\left(\xi_{0}, \cdots, \xi_{2 i+1}\right)$. Hence $c_{i+1} \in K\left(c_{i+2}, \cdots, \xi_{m+i+1}\right)$; also $A_{i+1}$ holds. Continuing, we have $c_{0}, \cdots, c_{m-1} \in K\left(c_{m}, \xi_{0}, \cdots, \xi_{2 m-1}\right)$. Hence $\xi_{0}, \cdots, \xi_{2 m-1}$ are algebraically independent over $K$. Thus $P$ is at least $2 m$-dimensional.

L.et $\Delta_{i}(\xi), i \geq m$, be the determinant of the coefficients of the forms $l_{0}(\xi), \ldots, l_{m-1}(\xi), l_{i}(\xi)$ regarded as linear forms in $c_{0}, \cdots, c_{m}$; that is,

$$
\Delta_{i}(\xi)=\left|\begin{array}{c}
\xi_{0} \cdots \xi_{m} \\
\xi_{1} \cdots \xi_{1+m} \\
\cdots \\
\xi_{m-1} \cdots \xi_{2 m-1} \\
\xi_{i} \cdots \xi_{i+m}
\end{array}\right|
$$

Then one finds $c_{j} \Delta_{i}(\xi)=0$, so that $\Delta_{i}(\xi)=0$. The coefficient of $\xi_{i+m}$ in this equation is a polynomial in the indeterminates $\xi_{0}, \ldots, \xi_{2 m-1}$; this coefficient contains the term $\xi_{0} \xi_{2} \cdots \xi_{2 m-2}$ and hence is not zero (therefore also $l_{0}(\xi), \cdots, l_{m-1}(\xi)$ are linearly independent over $\left.K(\xi)\right)$. Thus $P$ is at most $2 m$-dimensional, and hence exactly $2 m$-dimensional, Q.E.D.

LEMMA 3. Let $M=M(u)$ be the matrix:

$$
\left\|\begin{array}{c}
u_{0} \cdots u_{m} \\
u_{1} \cdots u_{1+m} \\
\cdots \\
u_{m} \cdots u_{2 m} \\
\cdots \\
u_{t} \cdots u_{t+m}
\end{array}\right\|, t \geq m
$$

Let $A$ be the ideal generated in $R_{t+m}$ by the $(m+1) \times(m+1)$ subdeterminants of $M(u)$. Then $A \subseteq P$.

Proof. Since $l_{0}(\xi), \ldots, l_{m-1}(\xi)$ are linearly independent over $K(\xi)$ (and in fact over any field containing $K(\xi))$ but $l_{0}(\xi), \ldots, l_{m-1}(\xi), l_{i}(\xi)$ are linearly dependent over $K(\xi)$, the matrix $M(\xi)$ has rank $m$. Hence $A \subseteq P$.

We want to prove $A=P$, in particular that $A$ is prime. Conversely, if we 
knew that $A$ were prime, we could conclude immediately that $A=P$. In fact, suppose $A$ is prime and let $\eta_{0}, \cdots, \eta_{t^{+m}}$ be a general point of $A$. Since $A$ has a basis of forms of degree $m+1$, no form of degree $m$ vanishes at $\eta$. Hence all $m \times m$ subdeterminants of $M(\eta)$ differ from zero, and it follows that $A$ is $2 m$ dimensional, whence $A=P$.

In proving $A=P$, we proceed by induction on $m$, the assertion being clearly true for $m=0$. For given $m$, we proceed by induction on $t(t \geq m)$. For $t=m$, we have to prove the following lemma.

Lemma 4. Let $D$ be the determinant

$$
\left|\begin{array}{c}
u_{0} \cdots u_{m} \\
u_{1} \cdots u_{1+m} \\
\cdot \cdot \\
u_{m} \cdots u_{2 m}
\end{array}\right|
$$

Then $D$ is different from zero and is irreducible in $R_{2 m}$.

Proof. By induction on $m$, being trivial for $m=0 . D$ is linear in $u_{0}$, the coefficient $\delta$ of $u_{0}$ being different from zero and irreducible by induction: in particular, therefore, $D \neq 0$. Also $D$ is linear in $u_{2 m}$ and the coefficient $\delta^{\prime}$ of $u_{2 m}$ is irreducible. $D$ is reducible if and only if $\delta$ is a factor of $D-u_{0} \delta$, hence of $D$. Similarly for $\delta^{\prime}$. Now $\delta$ and $\delta^{\prime}$ are not associates, since they are of different degree in $u_{0}$. So $D$ is reducible if and only if it is divisible by $\delta \delta^{\prime}$. For $m=1$, this means if and only if $u_{0} u_{2}-u_{1}^{2}$ is divisible by $u_{0} u_{2}$. This is not the case. For $m>1, D$ is reducible only if it is of degree at least $2 m$, whereas it is of degree $m+1$. Hence for every $m, D$ is irreducible.

Definition. An ideal is called homogeneous if it has a basis of forms. Similarly we call an ideal isobaric if it has a basis of isobaric polynomials.

Lemma 5. $A$ and $P$ are homogeneous and isobaric.

Proof. $A$ is clearly homogeneous. Moreover consider one of the $(m+1) \times$ $(m+1)$ subdeterminants of $M(u)$, say one involving the $i$ th and $j$ th rows, $i<j$. Then $u_{i+k-2}$ is the element in the $i$ th row and $k$ th-column and $u_{j+l-2}$ is the element in the $j$ th row and $l$ th column. Suppose $k>l$. The determinant in question has together with a term $\pi \cdot u_{i+k-2} u_{j+l-2}$ also a term $\pm \pi \cdot u_{i+l-2} \cdot u_{j+k-2}$, which is of the same weight. Hence if rows $i_{0}, \cdots, i_{m}$ are involved, each term has the weight of the term $u_{i_{0}} u_{i_{1}+1} u_{i_{2}+2} \cdots u_{i_{m}+m}$, that is, the determinant is 
isobaric. Thus $A$ is isobaric. As for $P$, we know that $p$ is homogeneous, and from this and the fact that $P=p \cap R_{t+m}$ one concludes immediately that $P$ also is homogeneous. To see that $P$ is isobaric, let $g(u) \in P$ and write $g(u)=$ $g_{r}(u)+g_{r+1}(u)+\cdots$, where $g_{j}(u)$ is zero or isobaric of weight $j$. It is clearly sufficient to prove $g_{r}(u) \in P$, assuming $g_{r} \neq 0$. Since $g(u) \in P$, we have

$$
h(c) g(u)=\sum A_{i}(c, u) l_{i}(c, u),
$$

where $h(c)$ is a polynomial in the $c_{i}$ alone, and the $A_{i}$ are polynomials in the $c_{i}$ and $u_{j}$. We assign to $c_{i}$ the weight $m-i$. Let $h(c)=h_{s}(c)+h_{s+1}(c)+\cdots$, where $h_{j}(c)$ is zero or isobaric of weight $j$ and $h_{s}(c) \neq 0$. Observe that the $l_{i}(c, u)$ are isobaric. Comparing terms of like weight on both sides of the above equation we see that $h_{s}(c) g_{r}(u)=\sum A_{i}^{\prime}(c, u) l_{i}(c, u)$. Hence $g_{r}(u) \in p$.

Theоrem 1. $A=P$. In particular, therefore, for $m>0, A: u_{0}=A$.

Proof. We proceed by induction on $m$ and $t$, and first show that $A: u_{0}=A$. Let $\xi_{0}, \cdots, \xi_{t^{+} m}$ be the general zero of $P$ introduced above. Let $D(u)$ be the determinant occurring in Lemma 4 . From $D(\xi)=0$ we see that $\xi_{2 m}$ can be written as a quotient of two polynomials in the indeterminates $\xi_{0}, \cdots, \xi_{2 m-1}$, with the denominator being

$$
\left|\begin{array}{ccc}
\xi_{0} & \cdots & \xi_{m-1} \\
\cdot & \cdot & \cdot \\
\xi_{m-1} & \cdots & \xi_{2 m-2}
\end{array}\right|
$$

which is irreducible by Lemma 4 . Hence we see that

$$
\left|\begin{array}{ccc}
\xi_{2} & \cdots & \xi_{m+1} \\
\cdot & \cdot & \cdot \\
\xi_{m+1} & \cdots & \xi_{2 m}
\end{array}\right| \neq 0
$$

(for were it zero, then $\xi_{2 m}$ could be written as a quotient of two irreducible polynomials in $\xi_{1}, \cdots, \xi_{2 m-1}$, the denominator this time not being an associate of the other denominator). Hence $\xi_{0}$ is algebraic over $K\left(\xi_{1}, \cdots, \xi_{t^{+} m}\right)$. Hence $\xi_{1}, \cdots, \xi_{t+m}$ defines a $2 m$-dimensional prime ideal $P_{1}$ in $K\left[u_{1}, \cdots, u_{t+m}\right]$; and $P_{1}$ is generated by the $(m+1) \times(m+1)$ subdeterminants of $M(u)$ which do not involve the first row of $M(u)$. Designating also by $P_{1}$, the extension of $P_{1}$ to $K\left[u_{0}, \cdots, u_{t+m}\right]$, we see that $P_{1} \subseteq A$. Let now $u_{0} g(u) \in A$. We write 
$u_{0} g(u)=\sum A_{i}(u) \Delta_{i}(u)$, where the $\Delta_{i}(u)$ are the $(m+1) \times(m+1)$ subdeterminants of $M(u)$, and the $A_{i}$ are polynomials. We write $A_{i}=A_{i}^{\prime}+u_{0} A_{i}^{\prime \prime}$, where $A_{i}^{\prime}$ does not involve $u_{0}$. We then have $u_{0}\left(g(u)-\sum A_{i}^{\prime \prime} \Delta_{i}(u)\right)=\sum^{i} A_{i}^{\prime} \Delta_{i}(u)$. The right hand side here is of degree at most one in $u_{0}$, hence $g_{1}=g(u)-$ $\sum A_{i} " \Delta_{i}(u)$ does not involve $u_{0}: g_{1}=g_{1}\left(u_{1}, \cdots, u_{t+m}\right)$. Now $g(u)$ and $\Delta_{i}(u)$ vanish at $\xi_{0}, \cdots, \xi_{m+t}$, hence so does $g_{1}$; that is, $g_{1}$ vanishes at $\xi_{1}, \cdots, \xi_{m+t}$. Hence, $g_{1} \in P_{1}$, whence $g \in A$. Hence $A: u_{0}=A$.

As a corollary to the above we get that $A: f=A$ for any polynomial $f \in R_{m+t}$ containing a term $d u_{0}^{r}, d \in K, d \neq 0(m>0)$. For suppose $f g \in A$ : to prove $g \in A$. We may suppose $f$ and $g$ isobaric; and also homogeneous. We then get $d u_{0}^{r} g \in A$, whence $g \in A$.

We proceed to prove that $A$ is prime. Let $\bar{l}_{i}=l_{i} / u_{0}=c_{0} v_{i}+\cdots+c_{m} v_{i+m}$, where $v_{i}=u_{i} / u_{0}$. We pass to the rings $\bar{R}_{t+m}=K\left[v_{1}, \cdots, v_{t+m}\right]$ and $\bar{S}_{t+m}=$ $K(c)[v]$. Observe that $v_{1}, \cdots, v_{t+m}$ are algebraically independent over $K$. Let $\bar{M}$ be the matrix of the coefficients of the $\bar{l}_{i}$, that is, the matrix:

$$
\left\|\begin{array}{ccccc}
1 & v_{1} & v_{2} & \cdots & v_{m} \\
v_{1} & v_{2} & v_{3} & \cdots & v_{1+m} \\
\cdot & & \cdot & & \cdot \\
v_{t} & v_{t+1} & v_{t+2} & \cdots & v_{t+m}
\end{array}\right\| \text {, }
$$

and let $A$ be the ideal generated in $R_{t+m}$ by the $(m+1) \times(m+1)$ subdeterminants of $M(v)$. Each such subdeterminant is a power of $u_{0}$ times an $(m+1) \times$ $(m+1)$ subdeterminant of $M(u)$; and vice-versa. It would therefore be sufficient to prove $\bar{A}$ prime, in fact it would be sufficient to prove that the extension of $A$ to the quotient ring $Q$ of $\bar{R}_{t+m}$ relative to the ideal $\left(v_{1}, \cdots, v_{t+m}\right)$ is prime. For suppose this proved and $g(u) h(u) \in A$, where we assume without loss of generality that $g(u), h(u)$ are homogeneous. Dividing by appropriate powers of $u_{0}$ and setting

$$
g(u) / u_{0}^{r}=\bar{g}(v), h(u) / u_{0}^{s}=\bar{h}(v)
$$

we get $\bar{g}(v) \bar{h}(v) \in \bar{A}$, whence by assumption $\bar{f}(v) \bar{g}(v)$ or $\bar{f}(v) \bar{h}(v)$, say $\bar{f} \bar{g}$ is in $\bar{A}$ for some $\bar{f}(v) \in \bar{R}_{t+m}, \bar{f} \notin\left(v_{1}, \ldots, v_{m}\right)$. Multiplying by a power of $u_{0}$ we find $u_{0}^{\rho} f(u) g(u) \in A$, where $f(u)$ contains a term $d u_{0}^{\sigma}$. Hence $g(u) \in A$.

The ideal $\bar{A}$ in $\bar{R}_{t+m}$ has $\xi_{1} / \xi_{0}, \ldots, \xi_{t+m} / \xi_{0}$ as a zero, hence is at least ( $2 m-1)$-dimensional. Also $\bar{A}$ remains at least $(2 m-1)$-dimensional upon extension to $Q$. In fact, if $\xi_{1} / \xi_{0}, \cdots, \xi_{t+m} / \xi_{0}$ determines $\bar{P}$ in $\bar{R}_{t+m}$, then 
$\bar{P} \subseteq\left(v_{1}, \cdots, v_{t+m}\right)$, as one sees from the fact that $\xi_{0}, \ldots, \xi_{t+m}$ determines a homogeneous and isobaric ideal $P$ and $u_{0} \notin P$.

Subtracting $v_{i}$ times the first row from the $(i+1)$ th row of $\bar{M}$, we get the matrix

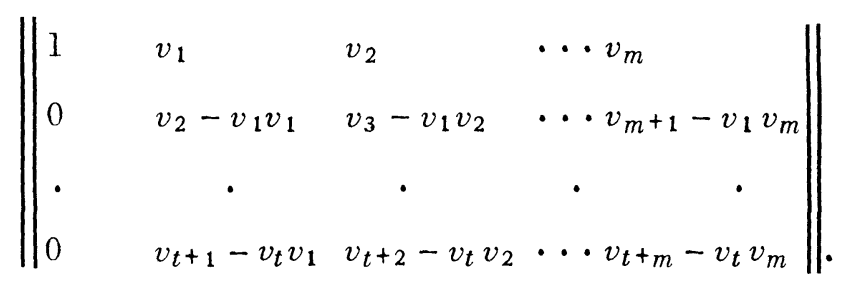

Each $(m+1) \times(m+1)$ subdeterminant of this matrix is also an $(m+1) \times$ $(m+1)$ subdeterminant of $M$. Hence one sees that every $m \times m$ subdeterminant of the matrix

$$
\left\|\begin{array}{cccc}
v_{2} & v_{3} & \cdots & v_{1+m} \\
\cdot & \cdot & \cdot & \cdot \\
v_{t+1} & v_{t+2} & \cdots & v_{t+m}
\end{array}\right\|
$$

is a leading-form of an element in $Q \cdot \bar{A}$. These $m \times m$ subdeterminants generate, by induction, a $2(m-1)$-dimensional prime ideal in $K\left[v_{2}, \cdots, v_{t+m}\right]$, and hence a $(2 m-1)$-dimensional prime ideal $\bar{q}$ in $K\left[v_{1}, \cdots, v_{t+m}\right]$. The leading form ideal of $\bar{A}$ contains or equals $\bar{q}$. If it contained $\bar{q}$ properly, it would be of dimension less than $2 m-1$. But an ideal and its leading form ideal have the same dimension [1; Satz 8]. Hence $\bar{q}$ is the leading-form ideal of $\bar{A}$ and $\bar{A}$ is $(2 m-1)$-dimensional.

Moreover $A$ is prime. For quite generally in a local ring, if an ideal $\bar{A}$ has a prime ideal $\bar{q}$ as leading form ideal, it must itself be prime. In fact, suppose $g h \in \bar{A}, g \notin \bar{A}, h \notin \bar{A}$. Then the leading form ideal $L F I(\bar{A}, g)$ of $(\bar{A}, g)$ contains $\bar{q}$ properly, and likewise for $(\bar{A}, h)$. But $\operatorname{LFI}(\bar{A}, g) \times \operatorname{LFI}(\bar{A}, h) \subseteq \operatorname{LFI}((\bar{A}, g) \times$ $(\bar{A}, h)) \subseteq L F I \bar{A}=\bar{q}$, a contradiction. Hence $\bar{A}$ is prime, and the proof is complete.

The following theorem is an immediate consequence of Theorem 1.

THEOREM 2. A basis for $\left[l_{0}\right] \cap K\{u\}$ is given by the $(m+1) \times(m+1)$ subdeterminants of the $\infty \times(m+1)$ matrix

$$
\left\|\begin{array}{cccc}
u_{0} & u_{1} & \cdots & u_{m} \\
u_{1} & u_{2} & \cdots & u_{\mathbf{l}+m} \\
\cdot & \cdot & .
\end{array}\right\| .
$$




\section{A. SEIDENBERG}

\section{REFERENCE}

1. W. Krull, Dimensionstheorie in Stellenringen, J. Reine angew. Math. 179 (1938), 204-226.

UNIVERSITY OF CALIFORNIA,

BERKELEY, CALIF ORNIA 


\section{PACIFIC JOURNAL OF MATHEMATICS}

\section{EDITORS}

\section{H.L. ROY DEN}

Stanford University

Stanford, California

E. HEWITT

University of Washington

Seattle 5, Washington

\section{R. P. DILWORTH}

California Institute of Technology

Pasadena 4, California

* Alfred Horn

University of California

Los Angeles 24, California

\section{ASSOCIATE EDITORS}

\begin{abstract}
H. BUSEMANN
HERBERT FEDERER

MARSHALL HALL
\end{abstract}

\section{P.R. HALMOS}

HEINZ HOPF

ALFRED HORN
R.D. JAMES

BØRGE JESSEN

PAUL LÉVY
GEORGE PÓLYA

J.J. STOKER

KOSAKU YOSIDA
UNIVERSITY OF BRITISH COLUMBIA CALIFORNIA INSTITUTE OF TECHNOLOGY UNIVERSITY OF CALIFORNIA, BER KELEY UNIVERSITY OF CALIFORNIA, DAVIS UNIVERSITY OF CALIFORNIA, LOS ANGELES UNIVERSITY OF CALIFORNIA, SANTA BARBARA MONTANA STATE UNIVERSITY

UNIVERSITY OF NEVADA

OREGON STATE COLLEGE

UNIVERSITY OF OREGON
UNIVERSITY OF SOUTHERN CALIFORNIA STANFORD UNIVERSITY UNIVERSITY OF UTAH WASHINGTON STATE COLLEGE UNIVERSITY OF WASHINGTON

AMERICAN MATHEMATICAL SOCIETY HUGHES AIRCRAFT COMPANY SHELL DEVELOPMENT COMPANY

Mathematical papers intended for publication in the Pacific Journal of Mathematics should be typewritten (double spaced), and the author should keep a complete copy. Manuscripts may be sent to any of the editors. Manuscripts intended for the outgoing editors should be sent to their successors. All other communications to the editors should be addressed to the managing editor, Alfred Horn, at the University of California Los Angeles 24, California.

50 reprints of each article are furnished free of charge; additional copies may be obtained at cost in multiples of 50 .

The Pacific Journal of Mathematics is published quarterly, in March, June, September, and December. The price per volume (4 numbers) is $\$ 12.00$; single issues, $\$ 3.50$; back numbers (Volumes $1,2,3$ ) are available at $\$ 2.50$ per copy. Special price to individual faculty members of supporting institutions and to individual members of the American Mathematical Society: $\$ 4.00$ per volume; single issues, $\$ 1.25$.

Subscriptions, orders for back numbers, and changes of address should be sent to the publishers, University of California Press, Berkeley 4, California.

Printed at Ann Arbor, Michigan. Entered as second class matter at the Post Office, Berkeley, California.

* During the absence of E.G. Straus.

UNIVERSITY OF CALIFORNIA PRESS - BERKELEY AND LOS ANGELES 


\section{Pacific Journal of Mathematics}

\section{Vol. 5, No. $4 \quad$ December, 1955}

Richard Horace Battin, Note on the "Evaluation of an integral occurring in servomechanism theory" ............................. 481

Frank Herbert Brownell, III, An extension of Weyl's asymptotic law for eigenvalues................................. 483

Wilbur Eugene Deskins, On the homomorphisms of an algebra onto Frobenius algebras .................................. 501

James Michael Gardner Fell, The measure ring for a cube of arbitrary dimension ....................................... 513

Harley M. Flanders, The norm function of an algebraic field extension. II ............................................ 519

Dieter Gaier, On the change of index for summable series ............ 529

Marshall Hall and Lowell J. Paige, Complete mappings of finite groups . . . . 541

Moses Richardson, Relativization and extension of solutions of irreflexive relations..................................... 551

Peter Scherk, An inequality for sets of integers .................. 585

W. R. Scott, On infinite groups ........................... 589

A. Seidenberg, On homogeneous linear differential equations with arbitrary constant coefficients ......................................... 599

Victor Lenard Shapiro, Cantor-type uniqueness of multiple trigonometric integrals...

Leonard Tornheim, Minimal basis and inessential discriminant divisors for a cubic field...

Helmut Wielandt, On eigenvalues of sums of normal matrices ... 